\title{
ROLE OF QUANTITATIVE ENDOTRACHEAL ASPIRATE AND CULTURES AS A SURVEILLANCE AND DIAGNOSTIC TOOL FOR VENTILATOR ASSOCIATED PNEUMONIA: A PILOT STUDY
}

\author{
SHALINI NAIR, NAGAMANI SEN, JOHN VICTOR PETER ${ }^{1}$, JOHN PRAKASH RAJ,
} K. N. BRAHMADATHAN²

\section{ABSTRACT}

BACKGROUND: Accurate diagnosis and appropriate treatment of ventilator associated pneumonia (VAP) is crucial for good outcomes. Endotracheal suctioning is performed in ventilated patients as part of routine care and for tracheal toileting. AIM: We evaluated if quantitative endotracheal aspirate (ETA) was a suitable alternative to bronchoalveolar lavage (BAL) for suspected VAP. In addition we assessed if surveillance ETA guided antibiotic selection for subsequent VAP. SETTING AND DESIGN: Prospective study in the surgical intensive care unit (ICU) of a tertiary hospital in India. MATERIALS AND METHODS: Two hundred consecutive patients with mean (standard deviation) APACHE II score of $12.3 \pm 5$ and requiring mechanical ventilation beyond 48 hours underwent surveillance ETA cultures. A second ETA and BAL were performed if the patient developed features of VAP. The threshold for microbiological diagnosis of VAP was taken as $10^{5}$ colony forming units $/ \mathrm{ml}$ (cfu/ml) for ETA and $10^{4} \mathrm{cfu} / \mathrm{ml}$ for BAL. STATISTICAL ANALYSIS: The sensitivity and specificity of surveillance and concurrent ETA aspirate cultures were compared with BAL cultures. RESULTS: VAP was suspected clinically and corroborated radiologically in $27 / 177$ patients (15.3\%). Although microbiological support for VAP was obtained by ETA in 19 patients, bronchoscopy was possible only in 13 patients, 8 of whom had isolates at significant threshold. Of the 16 organisms isolated from BAL, 11 were of significant threshold with 9/11 (82\%) BAL isolates having a similar antibiogram to a concurrent ETA. Only one BAL isolate (9\%), at significant threshold, was not isolated on a concurrent ETA. On the other hand just 6/11 BAL isolates (55\%) had an identical antibiogram to surveillance ETA. BAL had 3 additional isolates (27\%) at significant threshold not isolated on surveillance ETA. CONCLUSIONS: Concurrent quantitative ETA could substitute BAL cultures for VAP. Surveillance ETA at 48 hours of ventilation does not appear to assist with antibiotic selection for a subsequent VAP.

Key words: Antibiogram, bronchoalveolar lavage, quantitative analysis, surveillance endotracheal aspirate, ventilator associated pneumonia

Surgical Intensive Care Unit, ${ }^{1}$ Medical Intensive Care Unit, ${ }^{2}$ Department of Microbiology, Christian Medical College and Hospital, Vellore, India
Correspondence:

Dr. J. V. Peter,

Medical Intensive Care, Christian Medical College and Hospital,Vellore-632 004, India.

E-mail: peterjohnvictor@yahoo.com.au 


\section{INTRODUCTION}

Ventilator associated pneumonia (VAP) is the commonest intensive care unit (ICU) infection with an incidence ranging from 9 to $27 \%$ in intubated mechanically ventilated patients..$^{[1]}$ In the absence of a gold standard, VAP is assumed to be diagnosed more accurately by bronchoscopic sampling and microbiological cultures of the lower respiratory tract. ${ }^{[2]}$ Bronchoscopy, being invasive, is not uncommonly associated with complications, especially in patients on high respiratory supports. This has paved the way for less invasive tests such as endotracheal aspirates (ETA) and quantitative ETA cultures with a threshold of $10^{5}$ to $10^{6}$ bacteria per milliliter of exudates that is considered as optimal for the microbiological confirmation of VAP. ${ }^{[3]}$ More importantly, recent small trials have repeatedly shown that there is no advantage of bronchoscopic cultures over quantitative ETA cultures when mortality was considered as the end-point, ${ }^{[4-6]}$ further strengthening the case for quantitative ETA as a diagnostic tool.

Once VAP is suspected, early, aggressive, empiric therapy with broad-spectrum agents targeted at likely pathogens has been shown to be associated with lower mortality rates. ${ }^{[7-9]}$ Mortality is high even if an inappropriate empiric antibiotic is changed to a more appropriate one later in the course of therapy. ${ }^{[10]}$ Therefore the role of initial or subsequent surveillance cultures in assisting antibiotic selection in patients developing VAP assumes importance. This study was thus undertaken to assess: (a) if quantitative ETA cultures at clinical suspicion of VAP could substitute for the more invasive bronchoscopic cultures in the microbiological isolation of the organism and (b) if surveillance ETA at 48 hours of ventilation enabled appropriate choice of antibiotics for subsequent VAP.

\section{MATERIALS AND METHODS}

Patient selection: This study was conducted in the surgical intensive care unit (SICU) of a tertiary care teaching hospital in a developing country. All adults aged 18 years or more and mechanically ventilated for at least 48 hours and willing to participate in the study were considered for inclusion. Immunocompromised patients and those considered at high risk for subsequent bronchoscopy were excluded from the study. These high-risk patients included patients with bleeding diathesis, recent acute myocardial infarction, seizures, raised intra-cranial pressure and those on very high respiratory supports. It was felt that immunocompromised patients were a unique cohort in whom the differential diagnoses of alveolar densities were protean, and hence they were excluded from the study. The institutional review board approved the protocol, and written consent was obtained from the patients or their next of kin prior to inclusion in the study.

All patients staying beyond 48 hours were recruited to the trial and the demographic data was abstracted to specific data abstraction forms. Surveillance ETA was performed in all patients at the time of recruitment, (i.e.) after 48 hours of mechanical ventilation. These patients were carefully followed up for signs of VAP. This included, apart from clinical examination, regular recording of body temperature, observance of tracheal aspirate appearance, $\mathrm{PaO}_{2} / \mathrm{FiO}_{2}$ ratio, leukocyte count and chest radiographs. The diagnosis of VAP was based on the American College of Chest Physicians criteria ${ }^{[11]}$ and was defined as the occurrence of new and persistent radiographic infiltrates following intubation along with the presence of at least two of the following criteria: (a) temperature $>38.3^{\circ} \mathrm{C}$, (b) leukocytosis $>10,000$ cells $/ \mathrm{mm}^{3}$ and/or (c) purulent tracheo-bronchial secretions. Patients with clinical suspicion of VAP (based on the above criteria) underwent concurrent ETA and BAL with former sample collected first, lest it be diluted during BAL.

Procedure of ETA: It was performed under aseptic precautions using sterile suction catheters and traps. If the yield was $<1 \mathrm{ml}$, the procedure was repeated following chest physiotherapy. The presence of epithelial cells of $>10 \%$ implied contamination of the specimen whilst $<10 \%$ neutrophils suggested that the diagnosis of pneumonia was less likely. With quantitative analysis of ETA, the threshold for diagnosing VAP in this study was taken as $10^{5}$ colony forming units $/ \mathrm{ml}$ (cfu/ml).

Procedure of BAL: The procedure was carried out under aseptic precautions with adequate sedation and $\mathrm{FiO}_{2}$ of $100 \%$ through the endotracheal tube via a specific adaptor. No topical anesthesia or endobronchial suctioning was used during the advance of the bronchoscope. The scope was wedged into the orifice of the bronchus draining the segment likely to be involved, as judged radiologically, and the sample was collected after instilling three aliquots of $50 \mathrm{~mL}$ sterile saline. The sample was sent immediately for culture. The presence of $>1 \%$ squamous epithelial cells suggested a highly contaminated specimen. The microbiological threshold for the diagnosis of VAP was taken as $10^{4} \mathrm{cfu} / \mathrm{ml}$.

Method of quantitative analysis: Quantitative analysis of ETA was done according to gram stain smear interpretation. Depending on the number of organisms seen on direct smear, the clinical sample was diluted in 1 in 100 or 1 in 1000 and subsequently $10 \mu$ of diluted sample was uniformly inoculated on to blood agar, chocolate agar and McConkey agar. If no organism was seen on direct smear, an undiluted sample was inoculated on the agar plates. After overnight incubation the number of colonies were counted on each plate and multiplied by the appropriate dilution factor to express the colony count as cfu/ml. Samples with large mucus plugs were liquefied and homogenized by vortexing for one minute with glass beads followed by centrifuging at 3000 rotations per minute for 10 minutes. The cfu/ $\mathrm{ml}$ considered as significant in this study helps discriminate colonization from infection, with thresholds of $>10^{4} \mathrm{cfu} / \mathrm{ml}$ for BAL and $>10^{5} \mathrm{cfu} /$ $\mathrm{ml}$ for ETA being suggestive of infection rather than colonization. ${ }^{[12,13]}$

Statistics: Sample size was calculated based on the assumption that $85 \%$ of patients with VAP would be accurately detected by quantitative analysis of the ETA (with assumed rate of VAP being 14\%). This worked out as 200 patients assuming a difference of $5 \%$. Mann-Whitney $U$ test was performed to compare the ventilatory days between patients with or without VAP. The statistical analysis was carried out using SPSS 11.5.

\section{RESULTS}

The study was performed in the SICU over a 
1-year period. Although 200 patients underwent surveillance ETA at 48 hours of mechanical ventilation, only 177 patients could be followed up for the development of VAP [Figure 1]. Six patients were excluded because of an underlying immunocompromised state, 12 patients were discharged from the ICU against medical advice and 5 patients succumbed due to other causes. VAP was suspected clinically and corroborated radiologically in 27 of 177 patients $(15.3 \%)$ ventilated beyond 48 hours. Although 27 patients were initially suspected to have VAP based on the American College of Physicians criteria, ${ }^{[11]}$ bronchoscopy was performed in only 13 patients. In 8 patients, bronchoscopy had to be abandoned due to the development of significant hypoxemia or hypotension. Bronchoscopy was not performed in 5 patients as they were on high respiratory during the procedure despite the use of sedative agents [Figure 1]. Of the 13 patients who underwent bronchoscopy, 8 patients had isolates at significant threshold on BAL and fulfilled the microbiologic criteria for VAP of VAP were also fulfilled on ETA cultures in 11 of the 20 patients in whom bronchoscopy could not be performed [Figure 1].

Age and severity of illness (APACHE II score) supports and one patient was uncooperative [Table 2]. Microbiologic criteria for the diagnosis

did not predict the development of VAP in our cohort [Table 1]. However, female patients appeared to be at risk for the development of $\operatorname{VAP}(P=0.02)$. VAP occurred after a mean of $6.0 \pm 1.0$ days of ventilation. All but one patient

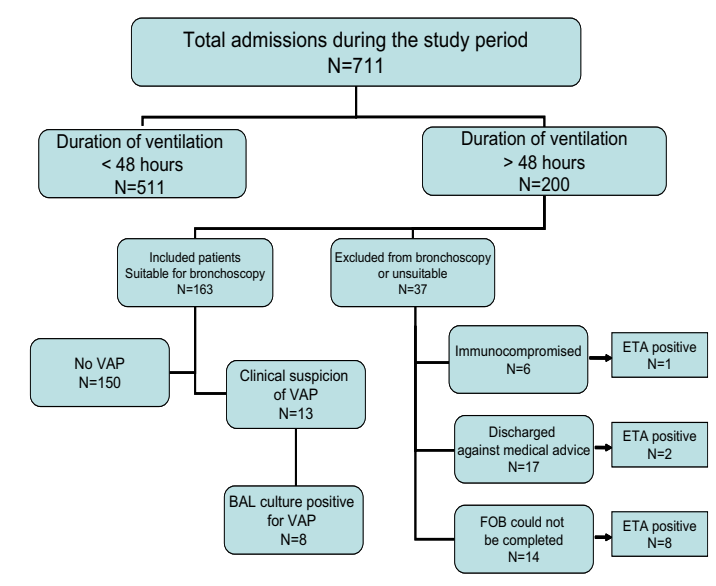

Figure 1: Flow chart of patient recruitment, inclusion and exclusion. Number of patients evaluated at each stage of the recruitment process and number of patients excluded from the trial with reasons for exclusion.

Among the patients who could not undergo bronchoscopy 14 had clinical suspicion of VAP. In this cohort eight patients in whom FOB was abandoned due to development of complications had ETA culture suggestive of VAP. Two patients who were discharged against medical advice had positive ETA culture and one of the immunocompromised patients fulfilled VAP criteria by ETA culture.

*Causes for exclusion from bronchoscopy: immunocompromised (6), uncooperative for FOB (1), high risk for FOB (5), procedure abandoned due to complications (8), discharged against medical advice (12), and died due to other reasons (5).

Table 1: Demographic data

\begin{tabular}{lccc}
\hline Variables & $\begin{array}{c}\text { Patients with ventilator } \\
\text { associated pneumonia } \\
N=13^{*}\end{array}$ & $\begin{array}{c}\text { Patients without ventilator } \\
\text { associated pneumonia } \\
N=163\end{array}$ & $P$ value \\
\hline Sex (M/F) & $6 / 7$ & $123 / 40$ & 0.02 \\
Age \pm SD (years) & $46.1 \pm 19.76$ & $42.84 \pm 14.99$ & 0.75 \\
APACHE II (mean \pm SD) & $13.6 \pm 6$ & $11.0 \pm 5$ & 0.83 \\
Ventilation duration & $10.0 \pm 5.35$ & $6.71 \pm 3.84$ & 0.03 \\
(mean \pm SD) days & &
\end{tabular}

Table 2: Microbiological data of patients with ventilator associated pneumonia

\begin{tabular}{|c|c|c|c|c|}
\hline $\begin{array}{l}\text { Organism in ETA } \\
\text { at } 48 \mathrm{~h}\end{array}$ & $\begin{array}{l}\text { Organism in ETA } \\
\text { at } n^{\text {th }} \text { day }^{t}\end{array}$ & $\begin{array}{l}\text { Organism in BAL } \\
\text { at } n^{\text {th }} \text { dayt }\end{array}$ & $n^{\text {th }}$ day $^{\dagger}$ & Final outcome \\
\hline $\begin{array}{l}\text { E. coli (S) } \\
\text { Klebsiella (S) }\end{array}$ & $\begin{array}{l}\text { Klebsiella } \\
\text { E. coli (S) }\end{array}$ & E. coli (S) & 7 & Discharge \\
\hline Klebsiella (S) & Klebsiella (S) & Klebsiella (S) & 5 & Discharge \\
\hline Pseudomonas & $\begin{array}{l}\text { Diphtheroids } \\
\text { Pseutomonas (S) }\end{array}$ & & 7 & Discharge \\
\hline Pseudomonas (S) & Pseudomonas & No growth & 6 & Death \\
\hline Enterobacter & Enterobacter & No growth & 2 & Death \\
\hline Pseudomonas (S) & $\begin{array}{c}\text { Pseudomonas (S) } \\
\text { Yeast }\end{array}$ & $\begin{array}{c}\text { Pseudomonas (S) } \\
\text { Yeast }\end{array}$ & 5 & Discharge \\
\hline Pseudomonas (S) ${ }^{*}$ & Pseudomonas (S) ${ }^{*}$ & Pseudomonas (S) & 7 & Death \\
\hline Staph aureus (S) & Staph aureus & Proteus (S) & & \\
\hline No growth & $\begin{array}{l}\text { Klebsiella (S)* } \\
\text { E. coli (S) }\end{array}$ & $\begin{array}{l}\text { Klebsiella (S)* } \\
\text { E. coli (S) }\end{array}$ & 10 & Death \\
\hline No growth & No growth & Pseudomonas & 6 & Death \\
\hline Pseudomonas (S) & Pseudomonas (S) & Pseudomonas (S) & 5 & Discharge \\
\hline No growth & Klebsiella & Acinetobacter & 6 & Discharge \\
\hline $\begin{array}{l}\text { Acinetobacter (S) } \\
\text { Alpha hem strep }\end{array}$ & Acinetobacter & Acinetobacter & 5 & Death \\
\hline Pseudomonas (S) & $\begin{array}{l}\text { Pseudomonas (S) } \\
\text { Acinetobacter }\end{array}$ & $\begin{array}{l}\text { Pseudomonas (S) } \\
\text { Acinetobacter }\end{array}$ & 23 & Death \\
\hline
\end{tabular}

$\mathrm{S}=$ Growth at significant thresholds, ${ }^{\mathrm{t}} \mathrm{n}^{\text {th }}$ day $=$ Day when VAP diagnosed, ${ }^{\circ}=$ identical antibiogram

developed late (> 5 days) onset VAP [Table 2]; in the patient who developed early VAP nosocomial pneumonia was diagnosed 48 hours after initiation of mechanical ventilation. The duration of ventilation, as expected, was longer in those developing VAP $(10.0 \pm 5.35$ versus $6.71 \pm 3.84, p=0.03$ ).

Microbiology: Gram negative organisms were the commonest causative agents identified by both ETA and BAL with a multi drug resistant pattern consistent with late onset VAP. Multi drug resistance was defined as a microorganism resistant to antibiotics considered "gold standard" for treatment of infection caused by that microorganism, or one that is only susceptible to antibiotics with more serious side effects than the standard ones. ${ }^{[14]}$ Isolates from a concurrent ETA, performed at the time of diagnosis of VAP, were compared with isolates obtained from a BAL. A total of 16 organisms were isolated from the BAL and
19 organisms from the concurrent ETA [Table 2]. In 2 patients no organisms were isolated from the BAL whilst no organism was isolated from a concurrent ETA in one patient. Of the 11 organisms identified in significant threshold in the BAL, 9 organisms were also identified in the significant threshold in the concurrent ETA and one at a lower threshold in the concurrent ETA. Nine of the 11 isolates on the concurrent ETA $(82 \%)$ had a similar antibiogram to the BAL isolates [Table 3]. BAL identified only one isolat at the significant threshold that was not isolated on a concurrent ETA (9\%). Three other isolates with a similar antibiogram were identified at non-significant thresholds in both the concurren ETA and BAL. Although a concurrent ETA identified 6 additional organisms that were not isolated on a BAL, only one was in the significan threshold. Thus if only isolates at a significan threshold were considered, then concurrent ETA compared with BAL has a sensitivity of $82 \%$, specificity of $91 \%$ and positive predictive value 
Table 3: Comparison of isolates obtained from a concurrent endotracheal aspirate and bronchoalveolar lavage performed at the time of diagnosis of ventilator associated pneumonia

Significant growth in endotracheal aspirate Significant growth ${ }^{+}$in BAL Non-significant growth or no growth in BAL Non-significant or no growth in endotracheal aspirate 9
2

Overall sensitivity $=82 \%$, Specificity $=91 \%$, Positive predictive value $=90 \%$

Concurrent endotracheal aspirate Vs BAL (expanded)

\begin{tabular}{|c|c|c|c|}
\hline & Significant growtht in BAL & Non-significant growth in $B A L$ & No growth in $B A L$ \\
\hline Significant growth ${ }^{\dagger}$ in ETA & $9^{+}$ & 0 & 1 \\
\hline Non-significant growth in ETA & $1^{+}$ & 3 & 5 \\
\hline No growth in ETA & 1 & 2 & 0 \\
\hline
\end{tabular}

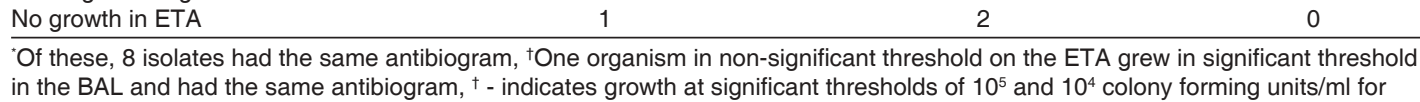
in the BAL and had the same
ETA and BAL respectively

of $90 \%$ in our cohort of patients.

On the other hand, of the 11 isolates grown in significant threshold in the BAL, only 6 organisms with an identical antibiogram (55\%) were isolated in the surveillance ETA performed at 48 hours of mechanical ventilation [Table 4]. Four isolates grown in significant thresholds in the surveillance ETA did not grow in the subsequent BAL. BAL had 3 additional isolates $(27 \%)$ at significant threshold not isolated on surveillance ETA [Table 2]. Thus the sensitivity of a surveillance ETA compared with BAL was $64 \%$ and specificity was $60 \%$ with a positive predictive value of $64 \%$.

When assessing ETA cultures in patients in whom bronchoscopy could not be performed at the time of diagnosis of VAP, 17 isolates were identified in 11 patients at significant threshold. Initial surveillance ETA at 48 hours of mechanical ventilation in these patients identified 9 isolates in 5 patients. Only two isolates had an identical antibiogram compared with the ETA at the time of diagnosis of VAP. Antibiotics were initiated based on ETAs taken at the time of diagnosis of VAP in these 11 patients. One patient did not complete treatment and left against medical advice. Three patients including an immunocompromised patient died whilst seven patients were discharged to the ward.

Table 4: Comparison of isolates obtained from surveillance endotracheal aspirate (ETA) and bronchoalveolar lavage (BAL)

\begin{tabular}{|c|c|c|}
\hline & Significant growth ${ }^{+}$in $B A L$ & Non-significant growth or no growth in $B A$ \\
\hline $\begin{array}{l}\text { Significant growth }{ }^{\dagger} \text { in ETA } \\
\text { Non-significant or no growth in ETA }\end{array}$ & $\begin{array}{l}7 \\
4\end{array}$ & $\begin{array}{l}4 \\
6\end{array}$ \\
\hline
\end{tabular}

Overall sensitivity $=64 \%$, Specificity $=60 \%$, Positive predictive value $=64 \%$

Surveillance ETA Vs BAL (expanded)

\begin{tabular}{lccc}
\hline & Significant growth ${ }^{\dagger}$ in $B A L$ & Non-significant growth in BAL & No growth in BAL \\
\hline Significant growth ${ }^{+}$in ETA & 7 & 1 & 3 \\
Non-significant growth in ETA & 1 & 0 & 2 \\
No growth in ETA & 3 & 4 & 0 \\
\hline Of these isolates only 6 isolates had the same antibiogram, ${ }^{+}$- indicates growth at significant thresholds of $10^{5}$ and $10^{4}$ colony
\end{tabular}

No growth in ETA

Of these isolates only 6 isolates had the sam
forming units/ml for ETA and BAL respectively

\section{DISCUSSION}

This study suggests that surveillance ETA was unable to assist in the appropriate identification of organism or choice of antibiotics in the majority of patients developing subsequent VAP. Only 6/11 BAL (55\%) isolates obtained at the time of diagnosis of VAP had a similar antibiogram to surveillance ETA at 48 hours of ventilation, limiting the value of routine surveillance ETA. These results are consistent with published literature elsewhere ${ }^{[15]}$ and CDC guidelines that restrict the role of routine surveillance of patients at high risk for health care associated pneumonia for specific clinical or epidemiologic or infection control objectives. ${ }^{[16]}$

The appropriate timing of surveillance ETA is unclear. ${ }^{[17-20]}$ Although the most proximate ETA prior to VAP may be best suited, overal it appears that surveillance ETA cultures have a limited role given the need for repetitive sampling of the respiratory tract as well as changes in the antibiogram that may occur with time.

The results comparing concurrent ETA and BAL performed at clinical suspicion of VAP appear promising with a sensitivity of $82 \%$ (for identical antibiogram) and specificity of $91 \%$. The Canadian Critical Care Trial group in a larger cohort reported similar results, but more importantly demonstrated that there were no significant differences in mortality, other clinical outcomes or the use of antibiotics between the two groups undergoing either an ETA or BAL as a diagnostic test. ${ }^{[21]}$ An earlier metaanalysis by Shorr et al, ${ }^{[22]}$ of four randomized trials of 628 patients, again suggested that invasive strategies do not alter mortality but affect antibiotic use and prescribing. Several other studies have again reported the utility and reliability of non-bronchoscopic methods in the diagnosis of VAP. ${ }^{[3,8,23]}$ This study, which has local relevance, had a design that incorporated the relevance of quantitative analysis of ETA (compared to BAL) as well as evaluating the role of surveillance ETA in subsequent VAP.

Given the current body of evidence reiterated by this study, routine use of BAL for the diagnosis and management of VAP cannot be justified particularly in the context of a developing country such as ours where added cost of BAL over quantitative ETA (Rs. 2442/ USD 57 for BAL culture and sensitivity vs Rs. 519/ USD 12 for ETA culture and sensitivity based on current costs) is passed on to the patient. Lack of expertise in performing bronchoscopy in several centers also limits the use of BAL as a routine diagnostic tool for VAP.

Several other aspects of our study ar worthy of mention. The incidence of VAP in our cohort was low (15.3\%) compared with results published elsewhere. This may be a reflection of a predominantly surgical cohor of patients as the medical, neurology, pediatric and cardio-thoracic patients are managed elsewhere in separate dedicated ICUs within the hospital. The low incidence may have also been helped by a very strict hand hygiene protocol that was rigidly implemented during the period of the study as part of an international study on nosocomial infections. That the incidence of pneumonia was highe amongst females was surprising and could not be explained, as other studies have not reported a sex bias for VAP, ${ }^{[24]}$ although female 
sex has been shown to be a mortality predictor in VAP. ${ }^{[24]}$

This study however has several important limitations. This was a single center study confined to one of the five ICUs within the institution. Further, with a much lower incidence of VAP than predicted, the effects of ETA were probably under demonstrated. Although the plan was to recruit 200 patients, the final numbers were only 163 due to the exclusion of several patients and the study had to be terminated due to limitation of resources and funding. That most of our patients received antibiotics (third generation cephalosporin) prior to ICU admission may also have affected the sensitivity pattern. Finally, though the admission APACHE II score was not high, 13 patients could not undergo fiberoptic bronchoscopy due to anticipated risk (5/13) or development of procedural risk (8/13) i.e. hypotension and hypoxemia, further limiting the number of patients who ultimately had bronchoscopic evaluation.

Notwithstanding these limitations, we submit that this pilot study from a tertiary centre in our country has added to our current knowledge and understanding of the role of ETA in the diagnosis of VAP as well as in the choice of antibiotic selection. This probably is the first study in our country that has evaluated both ETA at clinical suspicion of VAP and surveillance ETA at 48 hours of ventilation. There are several implications that arise from the study that are relevant to India. Foremost are the financial implications. Bronchoscopy is expensive, particularly in terms of initial investment costs. The cost of a bronchoscopic evaluation was Rs. 1900 (USD 45) in our study and this can add to the financial burden of a critically ill patient. Moreover, expertise is required for this procedure, which may not be readily available in many centers. The microbiological spectrum of isolates from this study is again more applicable to our country that sees a predominance of gram negative organisms causing VAP rather than methicillin-resistant Staphylococcus aureus. Larger trials from this part of the subcontinent would be invaluable in the design of protocols for diagnosis of VAP in cost and resource constrained developing nations.

\section{REFERENCES}

1. Chastre J, Fagon JY. Ventilator associated pneumonia. Am J Respir Crit Care Med 2002;165:867-903.

2. Heyland DK, Cook DJ, Marshall J, Heule M, Guslits B, Lang J, et al. The clinical utility of invasive diagnostic techniques in the setting of ventilator-associated pneumonia. Chest 1999;115:916-7.

3. Jourdain B, Novara A, Joly-Guillou ML, Dombret MC, Calvat S, Trouillet JL, et al. Role of quantitative cultures of endotracheal aspirates in the diagnosis of nosocomial pneumonia. Am J Respir Crit Care Med 1995;152:241-6.

4. Ruiz M, Torres A, Ewig S, Marcos MA, Alcón $A$, Lledó $R$, et al. Non-invasive versus invasive microbial investigation in ventilator associated pneumonia: Evaluation of outcome. Am J Respir Crit Care Med 2000;162:119-25.

5. Sole Violan J, Fernandez JA, Benitez AB, Cardenosa Cendrero JA, Rodriguez de Castro F. Impact of quantitative invasive diagnostic techniques in the management and outcome of mechanically ventilated patients with suspected pneumonia. Crit Care Med 2000;28:2737-41.
6. Sanchez-Nieto JM, Torres A, Garcia-Cordoba F, El-Ebiary M, Carrillo A, Ruiz J, et al. Impact of invasive and noninvasive quantitative culture sampling on outcome of ventilator-associated pneumonia: A pilot study. Am J Respir Crit Care Med 1998;157:371-6.

7. Rello J, Gallego M, Mariscal D, Sonora R, Valles $J$. The value of routine microbial investigation in ventilator-associated pneumonia. Am J Respir Care Med 1997;156:196-200.

8. Alvarez-Lerma F. Modification of empiric antibiotic treatment in patients with pneumonia acquired in the intensive care unit: ICU-acquired pneumonia study group. Intensive Care Med 1996;22: 387-94.

9. Luna CM, Vujacich $P$, Niederman MS, Vay C, Gherardi C, Matera J, et al. Impact of BAL data on the therapy and outcome of ventilatorassociated pneumonia. Chest 1997;111:67685.

10. Kollef MH, Ward S. The influence of mini-BAL cultures on patient outcomes: Implications for the antibiotic management of ventilatorassociated pneumonia. Chest 1998;113:41220.

11. Baselski VS, el-Torky M, Coalson JJ, Griffin JP. The standardization of criteria for processing and interpreting laboratory specimens in patients with suspected ventilator-associated pneumonia. Chest 1992;102:571S-9S.

12. Kirkpatrick MB, Bass JB Jr. Quantitative bacterial cultures of bronchoalveolar lavage fluids and protected brush catheter specimens from normal subjects. Am Rev Respir Dis 1989;139:546-8.

13. Pugin J, Auckenthaler R, Mili N, Janssens JP, Lew PD, Suter PM. Diagnosis of ventilator associated pneumonia by bacteriologic analysis of bronchoscopic and nonbronchoscopic blind bronchoalveolar lavage fluid. Am Rev Respir Dis 1991;143:1121-9.

14. Rello J, Paiva JA, Baraibar J, Barcenilla F, Bodi M, Castander D, et al. International conference for the development of consensus on the diagnosis and treatment of ventilator associated pneumonia. Chest 2001;120:955-70.

15. Delclaux C, Roupie E, Blot F, Brochard L, Lemaire $F$, Brun-Buisson C. Lower respiratory tract colonization and infection during sever acute respiratory distress syndrome: Incidence and diagnosis. Am J Respir Crit Care Med 1997;156:1092-8.

16. Tablan OC, Anderson LJ, Besser R, Bridges C Hajjeh R; CDC; et al. Guidelines for preventing health-care-associated pneumonia, 2003: Recommendations of CDC and the Healthcare Infection Control Practices Advisory Committee. MMWR Recomm Rep 2004;53:1-36.

17. Michel F, Franceschini B, Berger P, Arnal JM Gainnier M, Sainty JM, et al. Early antibiotic therapy for BAL confirmed ventilator associated pneumonia: A role for routine endotracheal aspirate cultures. Chest 2005;127:589-97.

18. Hayon J, Figliolini C, Combes A, Trouillet JL, Kassis N, Dombret MC, et al. Role of seria routine microbiologic culture results in the initial management of ventilator-associated pneumonia. Am J Respir Crit Care Med 2002;165:41-6.

19. Rajasekhar T, Anuradha K, Suhasini T, Lakshm $V$. The role of quantitative cultures of nonbronchoscopic samples in ventilator associated pneumonia. Indian J Med Microbiol 2006;24 107-13.

20. Dennesen PJ, Van der Ven AJ, Kessels AG, Ramsay G, Bonten MJ. Resolution of infectious parameters after antimicrobial therapy in patients with ventilator-associated pneumonia. Am J Respir Crit Care Med 2001;163:1371-5.

21. Canadian Critical Care Trials Group. A randomized trial of diagnostic techniques for ventilator-associated pneumonia. N Engl J Med 2006;355:2619-30.

22. Shorr AF, Sherner JH, Jackson WL, Kollef $\mathrm{MH}$. Invasive approaches to the diagnosis of ventilator associated pneumonia: A meta- 
analysis. Crit Care Med 2005;33:46-53.

23. Wu CL, Yang Dle, Wang NY, Kuo HT, Chen PZ. Quantitative cultures of endotracheal aspirate in the diagnosis of ventilator associated pneumonia in patients with treatment failure. Chest 2002;122:662-8.

24. Combes A, Luyt CE, Fagon JY, Wolff M,
Trouillet JL, Chastre J. Early predictors for infection recurrence and deaths in patients with ventilator associated pneumonia. Crit Care Med 2007;35:146-54.

Source of Support: Nil. Conflict of Interest: None declared. 